\title{
Introduction to IT Enabled Collaboration for Development Minitrack
}

\author{
Xusen Cheng \\ University of International \\ Business and Economics, \\ Beijing, China \\ xusen.cheng@uibe.edu.cn
}

\author{
Xiangbin Yan \\ University of Science \& \\ Technology Beijing, \\ Beijing, China \\ xbyan@ustb.edu.cn
}

\author{
Deepinder Bajwa \\ Western Washington University \\ Bellingham, USA \\ deepinder.bajwa@wwu.edu
}

Over the past three decades, collaboration technologies have been applied in different countries in many global regions to support team work. Developed regions such as Western Europe and North America have paved the way, pioneering the use collaboration technologies to successfully support team work. Trends towards globalization provide opportunities for organizations in developing regions such as the Eastern Europe, Asia, Africa, and South America to adopt collaboration technologies to improve the effectiveness and efficiency of team work in face-to-face and virtual settings within and across organizations.

In the fourth year of this mini-track, we changed the name of the minitrack slightly, and we accepted four papers for inclusion in the HICSS conference proceedings. All the papers submitted were peer reviewed by knowledgeable scholars.

The first paper is titled "Operational mechanism of digital humanistic crowdsourcing project based on actor network theory" by Xiaojuan Xu, Hui Gao, Jianshan Sun, Cuicui Wang, and Wei Xu. In their paper, the authors aim to promote the development of digital humanity-related crowdsourcing projects based on actor network theory. Data was collected from different sources, including project development documents and semi-structured interviews. Their findings suggest that task guidance, user motivations, platform designs, and competition evaluations are the main obstacles preventing actors to engage in crowdsourcing projects. These findings will serve as a base for future studies in this area.

The second paper is titled "The role of collaboration between incumbent firms and start-ups on customers' adoption of digital innovation" by Nihal Islam and Peter Buxmann. Digital technology is an important factor for a company to enter into markets. Based on analyses of qualitative data collected from 16 customer interviews, this study offers valuable insights into how collaboration between incumbent firms and start-ups should be presented to customers of digital innovations. Their analyses provide interesting insights into costumers' subjective stance towards adoption of digital innovations.

The third paper is titled "Does daily travel pattern disclose people's preference?" by Mingxin Gan, Ling Gao, and Yang Han. People's characteristics and preference have attracted attention in both research and practice. This paper attempts to capture people's characteristics from their daily travel pattern. The authors deploy statistical procedures and incorporate historical trajectories with real-time positions from a location-based social network. Their findings suggest that people's preference can be inferred from the developed activity and colorfulness measures.

The fourth paper is titled "Can process facilitation improve globally distributed collaboration? An action design research" by Xusen Cheng, Shixuan Fu, Jianqing Huang, and Gert-Jan de Vreede. In their paper, the authors design a process facilitation support application and propose two artifacts including: facilitated collaboration process and collaborative tools for applications of process guidance. The study provides important insights into globally distributed collaboration and business context.

These four papers all focused on research frontier in information technology including: globally distributed collaboration, digital humanity-related crowdsourcing projects, collaboration between incumbent firms and start-ups, and people's characteristics and preference. Based on survey, case studies and design science, these papers contribute to the area of IT-enabled collaboration for development. Each of the four papers provided implications for practice.

In conclusion, we would like to thank all the authors who submitted their papers to our mini-track and all the reviewers that provided valuable feedback to the authors of submitted manuscripts. As we move forward, we hope to attract more research papers from the developing regions of the world from researchers who are interested in IT-enabled collaboration for development. 